\title{
Reversed-flow models
}

\author{
Tomas Skoglund ${ }^{\mathrm{a}}$ \\ ${ }^{\mathrm{a}}$ Tetra Pak Processing Systems \\ Ruben Rausings gata, SE-221 86 Lund, Sweden \\ tomas.skoglund@tetrapak.com
}

\begin{abstract}
Component models for bidirectional flow in process lines were developed to account for propagation of fluid properties and balance equations for mass, heat and momentum. The models performed well in simulations with flow in arbitrary direction. The models increased the stability in simulations of cases with short, unintentional reversed flow.
\end{abstract}

Keywords: reversed flow; bidirectional flow; process line; property propagation; balance equations; fluid composition; mass fraction; liquid food

\section{Introduction}

Fluid flow accounting for mass, thermal and momentum balances have been modelled in Modelica [6] and was reported in e.g. [9] (Eborn, 2001), [10] (Tummescheit, 2002) and [11] (Casella and Leva, 2003). The latter paper also addressed flow reversal.

A special case is liquid food process lines, e.g. lines for production of UHT milk in dairies. Dynamic simulation of this has been practiced during some years by means of a Modelica-based dynamic model library [1] (Skoglund, 2003), [2] (Skoglund and Dejmek, 2006), [5] (Skoglund, 2007) and [12] (Gäfvert et al, 2008). Beside the fundamental laws of conservation, e.g. mass and heat, the model library addressed particular characteristics of liquid food process lines. For example dynamic propagation of fluid properties was considered, [3] (Skoglund and Dejmek, 2006), due to the need of simulating start-up and shut-down with fluid changes, which are occurring frequently in the addressed applications.

Also typical for liquid food process lines is that flow directions in many cases are reversed. As fluid propagation has to be considered simultaneously, it has to be addressed in the models for correct mass and thermal balance equation, which otherwise may lead to non-physical behaviour and eventually cause a crash of the simulation. This article describes how the model library was adapted for reversed flow.

\section{The "FoodProcessing" library}

Since the start of the development of the "FoddProcessing" library [1] much more work was spent to address characteristics of liquid food process lines. Thus a model for axial-dispersed plug flow (ADPF) was derived [3] and some models were extended for reaction kinetics [8]. Figure 1 shows the library "FoodProcessing" in the Modelica tool Dymola [7], which was also described in earlier publications, [1], [2] and [12].

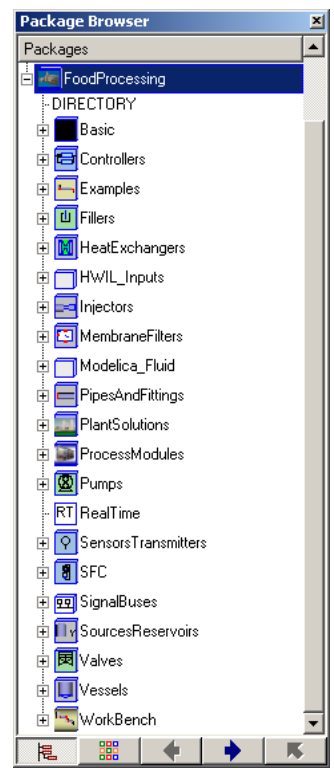

Figure 1. The "FoodProcessing" library, which has been described earlier in [1], [2] and [12]. 
The library has since been used to configure many process lines to test various performances, e.g. product losses. Thus the arise, development and propagation of mixing zones was simulated for product filling and emptying in a commercial UHT line for milk sterilization [4] (Skoglund \& Dejmek, 2007). The library was also used for trouble shooting and testing new design ideas as regards both process design and control algorithms. Furthermore simulation for functional test of complete process lines with the actual control system was enabled through further library development. Hence the library was adapted for real-time Hardware-in-theloop simulation (HILS) [12]. Software for signal communication with the control system was developed too.

\section{Reversed-flow processes}

In some processes the direction of the fluid flow will vary as the plant state shifts. Figure 2 shows a typical case where different flow directions are normal at different plant states, e.g. filling and emptying of product (food medium) to/from a tank.

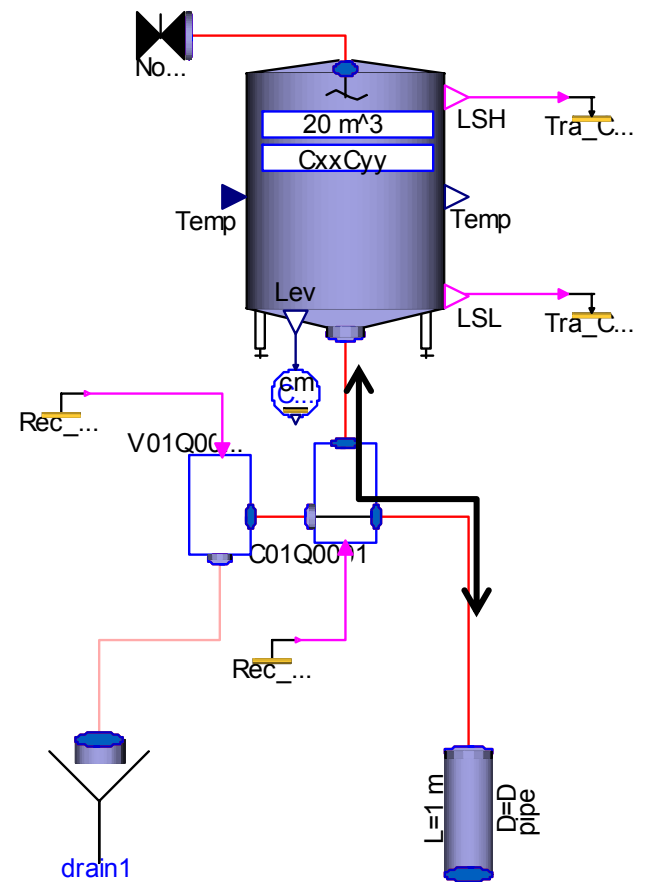

Figure 2. A typical case with two flow directions. The black, bold arrows show the product (food medium) flow at filling and emptying of the vessel.

In volumes and junctions mass and heat balances require that the properties from the correct connections are used in the balance equations. In Figure 2 this is valid for the tank and three-port valve at the bottom of the tank. Furthermore, in fluid channels (e.g. the pipe in Figure 2) it is important that fluid properties from the correct connection are used for the dynamic momentum balances and friction forces.

\subsection{Transients - unintentional reversed flow}

In many situations the flow direction should normally never change. However due to transients during valve transitions, the flow direction might be reversed for a short period of time, This might be enough to cause simulation problems if reversed flow is not handled adequately in the models. Figure 3 shows an example of that

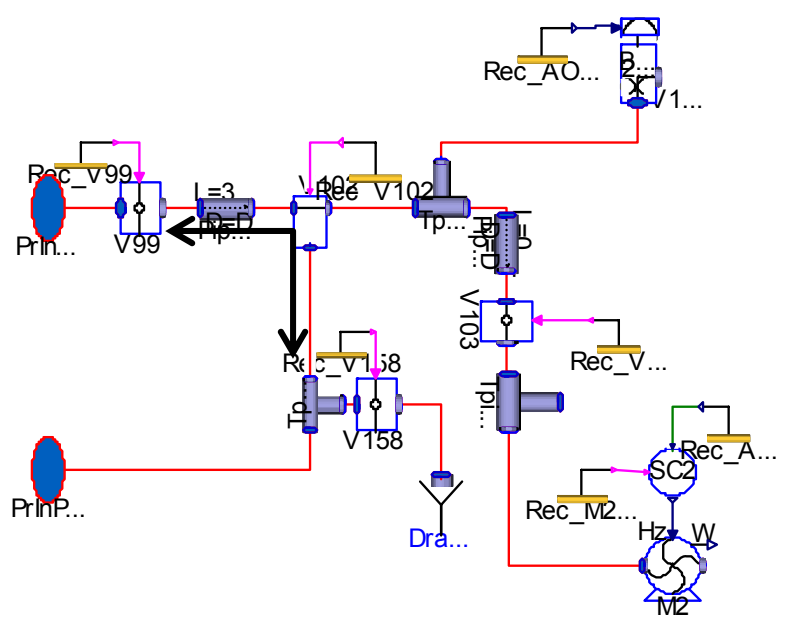

Figure 3. Example of case where the flow direction may be reversed just during state transition in a plant. The black, bold arrows indicate two flow directions, which both deviate from the normal.

\subsection{The problem}

The problem arises when flow direction and fluid properties not match. The problem occurs in balance equations for mass and heat. To illustrate the problem we can study a simple mass balance in a volume with one port for normal flow in and one port for normal flow out of a component. We can assume an incompressible fluid with constant density flowing through the constant volume, $V$, with a positive constant flow rate, $Q$. This yields the following mass balance for a certain fluid component with the mass fraction $X$ in the volume, thus also flowing out from the volume.

$V \frac{\partial X}{\partial t}=\left(X_{\text {in }}-X\right) Q$

Eq. 1

$X_{i n}$ is the mass fraction into the component through the normal input port. With all variables and constants positive, Eq. 1 describes a stable system where 
$X$ approaches $X_{i n}$. If the flow rate changes direction, Eq. 1 changes character to Eq. 2, which describes an incorrect and unstable system.

$$
V \frac{\partial X}{\partial t}=\left(X-X_{\text {in }}\right)|Q|
$$

The correct conversion of Eq.1 would be

$$
V \frac{\partial X}{\partial t}=\left(X-X_{\text {out }}\right) Q=\left(X_{\text {out }}-X\right)|Q| \quad \text { Eq. } 3
$$

$X_{\text {out }}$ is the mass fraction into the component through the normal output port. This conditional rearrangement of the equations must be handled when the flow changes sign.

\section{Reversed-flow models}

The fundamental idea with the reversed-flow models presented in this paper is to use a record containing fluid properties declared as input and output in the connectors. Hence there is a need for two types of connectors "In" and "Out" to match the input with an output and the output with an input. This signal-based approach was suggested by Casella and Leva [11]. Thus the connectors must be connected in pairs "In" to "Out". Therefore all components are modelled for a "nominal" flow direction. The consequence is that some component models have to be made in more than one version to match different nominal flow directions. One example of that is the three-port valve in Figure 2, a valve which in some applications normally splits the flow and in other applications normally merges two flows. Another example is the T-pipes in Figure 3, where two of them normally split the flow whereas one of them normally merges two flows.

\subsection{Modelica code for connectors}

A Modelica code for the nominal flow direction "into" a component is given below.

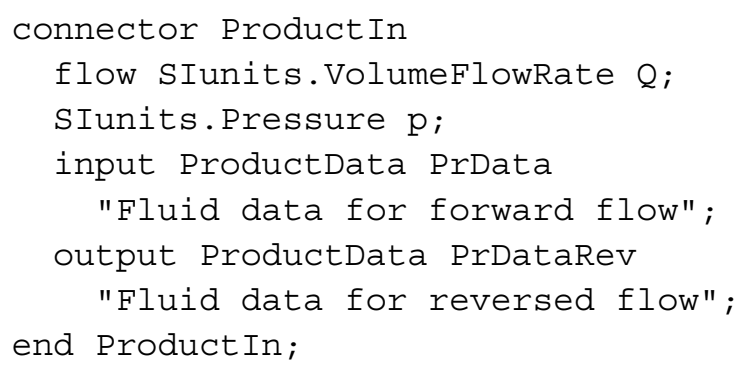

In this example the thermal properties are not declared as a pair of flow and cross variables. The fluid properties are declared in the record ProductData. In [11] the input and output handled the specific enthalpy. In this case they include not only thermal properties, but also density and mass fraction properties for the fluid composition as this is important to consider in many liquid food cases (see e.g. [3])

Corresponding to the above connector is a connector for the nominal flow direction "out of" a component. The Modelica code is given below.

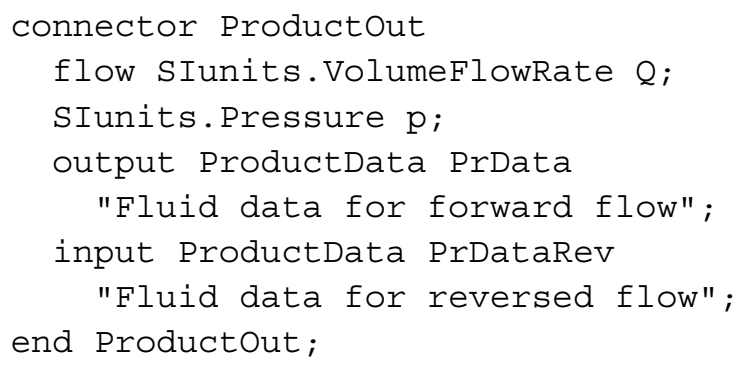

\subsection{Conditional equations}

As the flow direction alters, the fluid properties used in the equations, e.g. mass fraction balance, must swap the used data from the connectors. The following Modelica code shows the principle used in a constant volume in which the fluid (with the density rho) is mixed ideally (instantly).

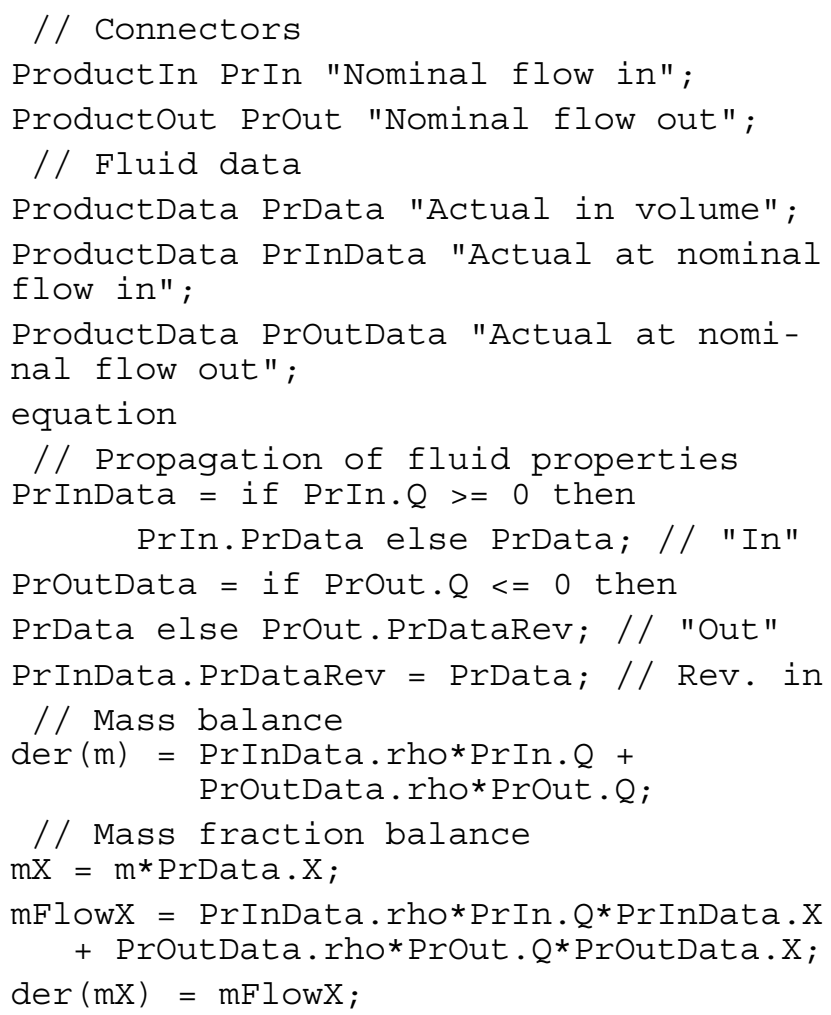




\section{Sensors for reversed flow}

System simulations mostly need sensor models, Thus, in a system where the fluid may flow in either of the two directions, sensors for fluid properties must account for that, i.e. the property (e.g. concentration of a fluid component) must account for the flow direction. A sensor adaptor was modelled to pick up fluid properties from the flow path. A part of the code for that adaptor is given below.

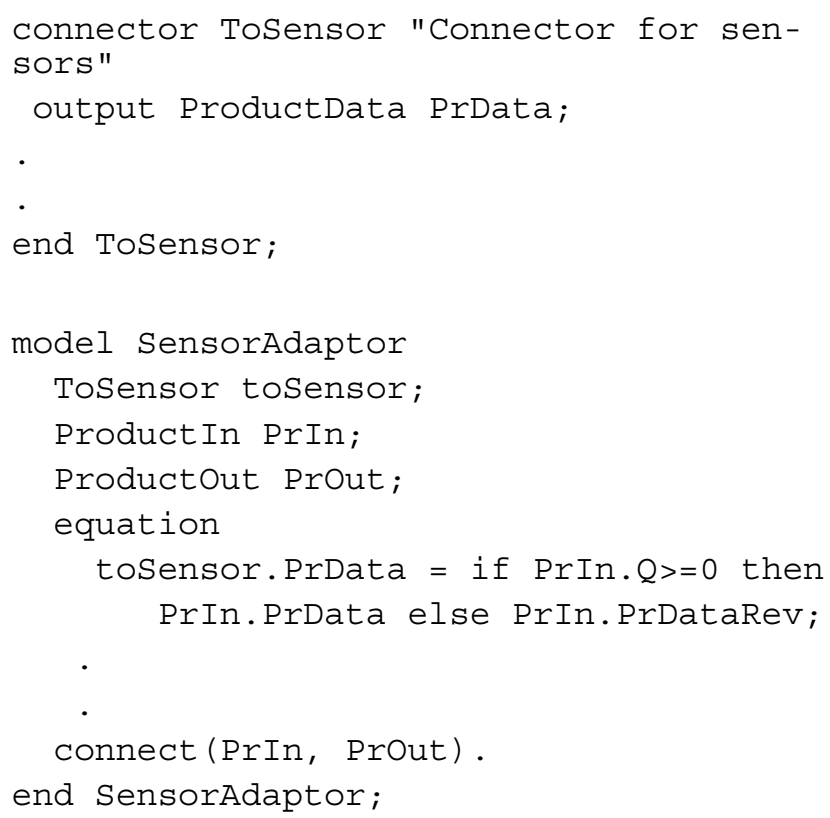

The sensor models have a corresponding connector to the ToSensor to pick up the fluid properties.

\section{Process examples and results}

As already mentioned, reversed flow models are required both to get correct simulation results in systems where reversed flow takes place normally, and to get stable simulations in situations with short, unintentional reversed flow.

\subsection{Normal situations with reversed flow}

A simple example illustrating the solution of reversed flow models is shown in Figure 4. The system comprises two pressure tanks, a pipe, a centrifugal pump and two sensors for the concentration of a fluid component. (In this case the sensor adaptors are incorporated in the pipe.) Tank 1 has an initial content of $10 \mathrm{~m}^{3}$ with a concentration of $10 \%$ carbohydrates, while Tank 2 has an initial content of $2 \mathrm{~m}^{3}$ with $0 \%$ concentration. The pump M1 runs intermittently 250 seconds with a stop time equal long. During the pumping the fluid volumes in the vessels will change. The levels and gas pressures will change accordingly. The consequence is that after a while the flow will go in the reversed direction during the periods when the pump is stopped.

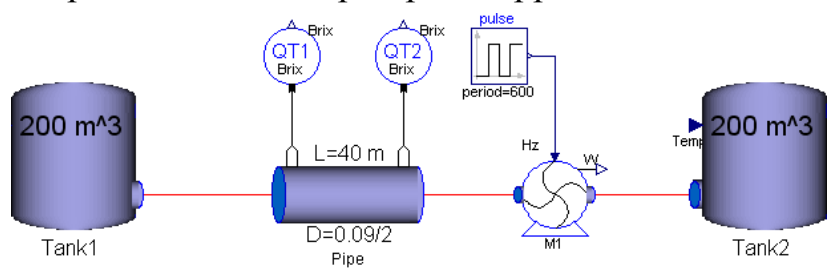

Figure 4. Example of simple system with forward and reversed flow

The pipe model is based on mass balance as axially dispersed plug flow (Eq. 4), and momentum balance (Eq. 5) in an incompressible fluid.

$$
\begin{array}{ll}
\frac{\partial C}{\partial t}+v \frac{\partial C}{\partial x}-D \frac{\partial^{2} C}{\partial x^{2}}=0 & \text { Eq. } 4 \\
\frac{\partial(\rho v)}{\partial t}+\frac{\partial p}{\partial x}+p_{f}+\rho g \frac{\partial z}{\partial x}=0 & \text { Eq. } 5
\end{array}
$$

Explanations of symbols are given in section 8, Notation. Figure 5 shows the simulation result. Note; When the fluid changes flow direction there is a time lag - due to the transportation through the pipe until the "new" fluid composition reaches the sensor at the current pipe exit. The sensors are modelled as ideal sensors with no dynamics.

Another more realistic example, though still simplified, is shown in Figure 6. It shows a milk supply, a valve cluster and two tanks. Figure 7 shows the simulation result of tank filling with milk, milk transport from tank to tank, and water flush of a tank.

\subsection{Reversed flow during transitions - stability}

The example in Figure 8 is used to illustrate the problem with instability in situations with short periods of reversed-flow without accounting for it. The example was run both without and with models accounting for reversed flow.

In the valve $\mathrm{V} 1$ the normal flow direction is from the sources of water and juice concentrate to the out port on the right hand side. The valve is of type change-over and connects either of the two ports from the sources of water or juice concentrate to the out port. During the valve transition (after $10 \mathrm{~s}$ ), all three ports were connected to each other for a short moment of time and, due to the pressures and flow dynamics, the flow was reversed for about $1 \mathrm{~s}$ in the valve port for water. The simulation results are shown in Figure 9. In the case of models not accounting for 
the reversed flow, the simulation crashed after approximately $11.5 \mathrm{~s}$ (1.5 s after the valve activation). The reason is the incorrect balance equations, due to the reversed flow of water, which cause the nonphysical increase of temperature and sugar concen- tration, which can be seen in the lower graphs of Figure 9. In the case with models accounting for the reversed flow, the temperature and sugar concentration were correct and the simulation did not crash.
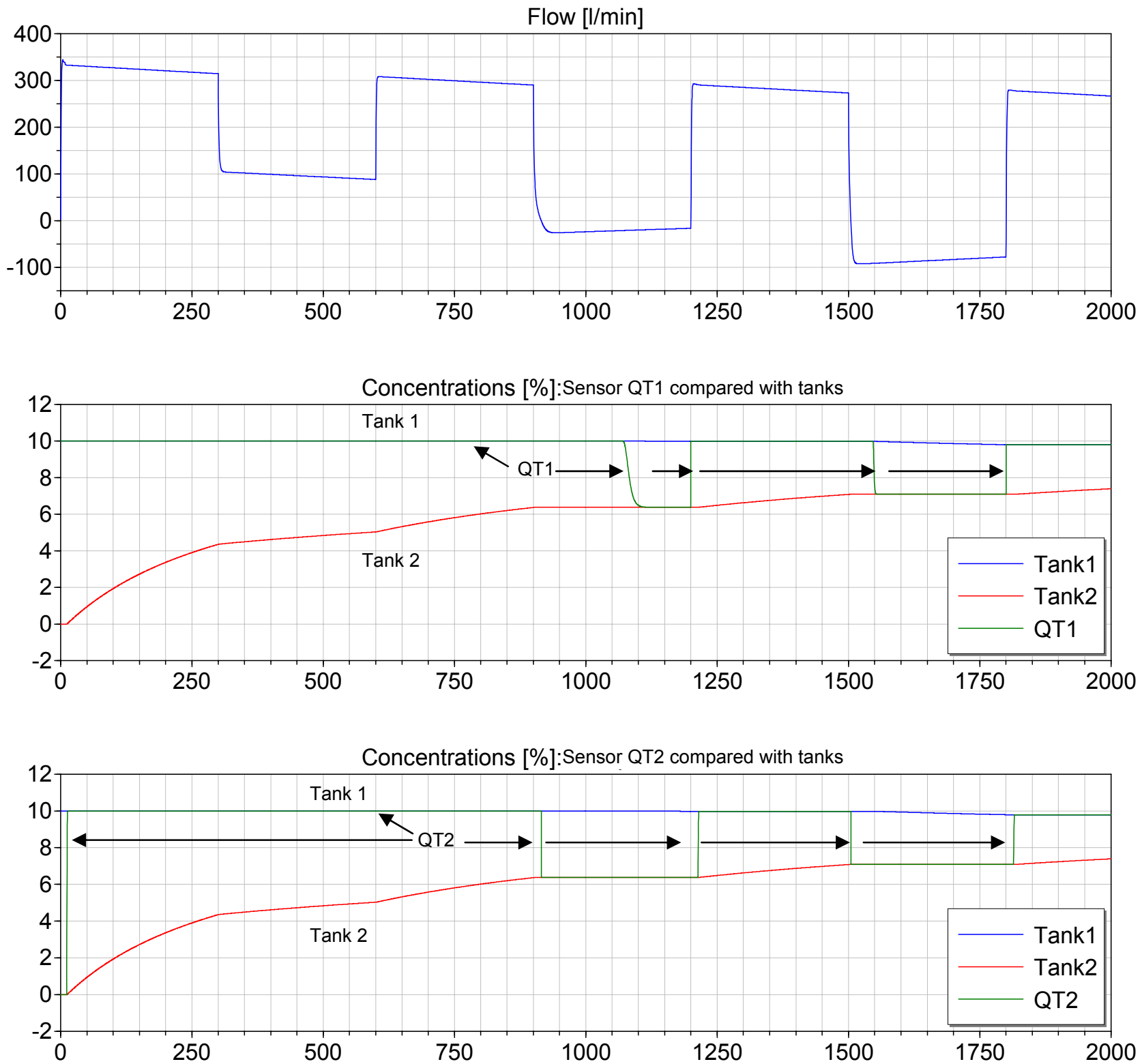

Figure 5. Simulation result of system shown in Figure 4. The time scale is in seconds. The concentrations in the tanks are shown in both middle and bottom figures to facilitate comparison. The locations of the sensors QT1 and QT2 are shown in Figure 4. Note; When the fluid changes flow direction there is a time lag - due to the transportation through the pipe - until the "new" fluid composition reaches the sensor at the current pipe exit. (For clarity in black/white print, some curves are marked directly in the graphs as a complement to the legends.) 


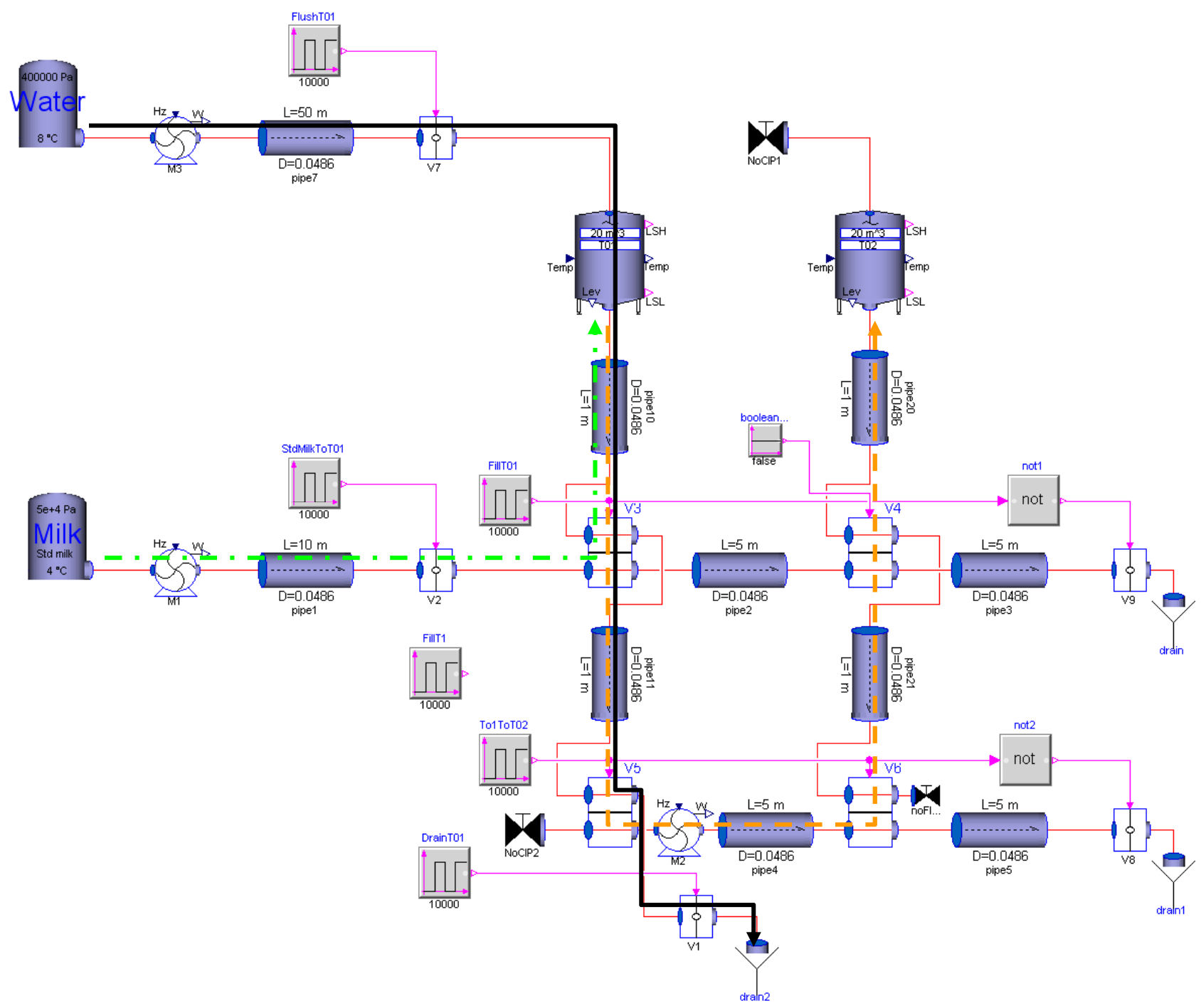

Figure 6. A typical, though simplified, valve cluster (the four valves: V3, V4, V5, V8) with two tanks. A sequence of three steps is run through. 1: (green, bold, dash dotted arrow) Milk is pumped to tank T01. 2: (orange, bpld, dashed arrow) The milk is pumped from tank T01 to tank T02. 3: (black, solid, bold arrow) Tank T01 is flushed with water to drain. During these three phases fluids are running in two directions through the tank T01, the valve V3 and the pipe pipe10. Simulation results are shown in Figure 7. 

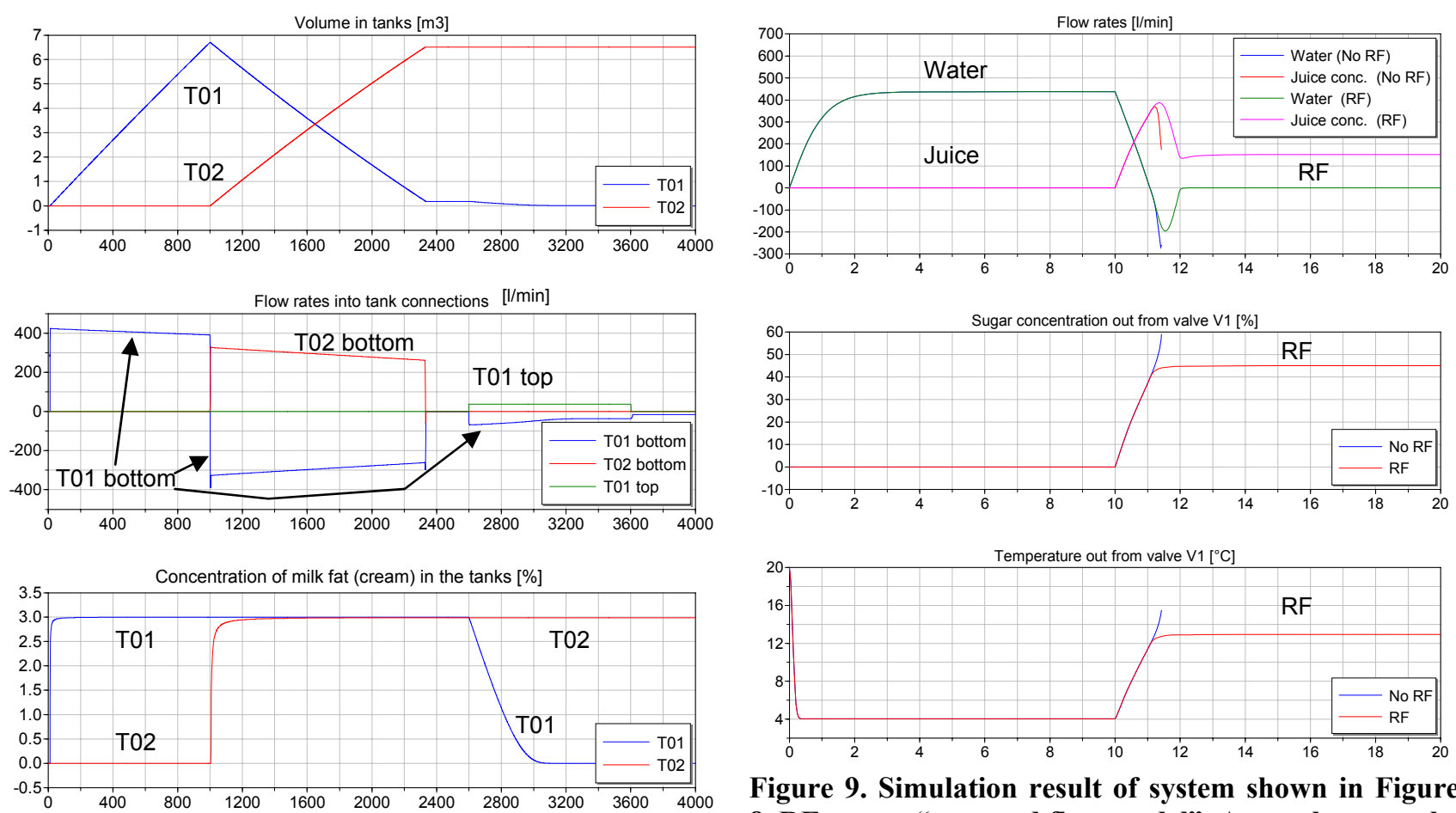

Figure 7. Simulation result of system shown in Figure 6. The time scale is in seconds. (For clarity in black/white print, the curves are marked directly in the graphs as a complement to the legends.)

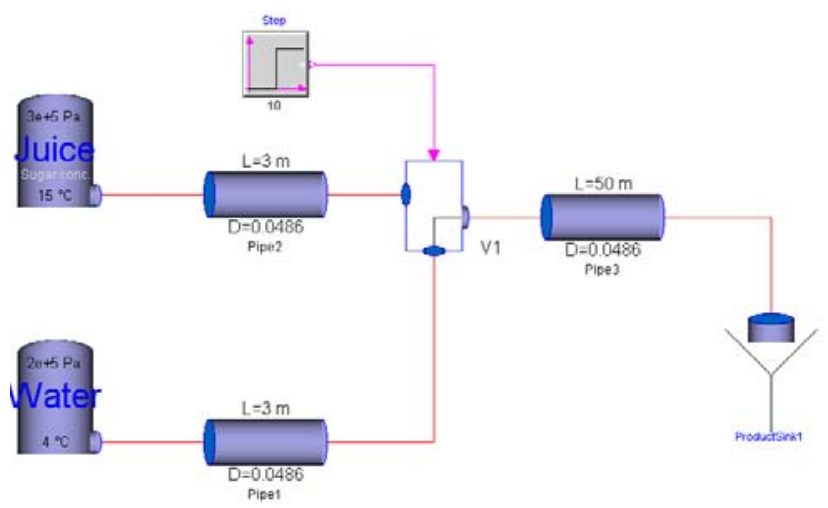

\section{Conclusions}

Fluid flow is often intentional bidirectional. In other cases, in spite of the intended unidirectional flow, unintentional reversed flow may occur during short periods of time. Therefore, models accounting for bidirectional fluid flow are required in many applications. Models accounting for this must address all properties propagating along the $1 \mathrm{D}$ flow coordinate, and the fact has to be considered, that the properties will differ in a connection point of a

Figure 8. Simple model to illustrate problem with instability due to short, unintentional reversed flow. In the valve $\mathrm{V} 1$ the normal flow direction is from the sources of water and juice concentrate. The valve is a change-over type and connects either of the two ports from the sources of water or juice concentrate to the output. During the valve transition (at $10 \mathrm{~s}$ ), all three ports were connected to each other for a short moment of time, which in this case caused a short moment of reversed flow of water component depending on the flow direction in each moment of time.

The modelling problem is twofold; the connector problem and the balance equation problem. In this paper the solution was based on the input/output connector principle with conditional use of connector data in the heat balance, [11]. A solution, which also includes mass fraction balances, was demonstrated in this paper. Furthermore, models to adapt sensor models for arbitrary flow direction was proposed and demonstrated.

Correct and stable simulations were achieved for flow in arbitrary direction during simulations of fluid flow systems particularly relevant for liquid food applications. 


\section{Notation}

C Volumetric concentration, $\mathrm{kg} / \mathrm{m}^{3}$

$D$ Dispersion coefficient in axial direction, $\mathrm{m}^{2} / \mathrm{s}$

g Constant of gravity acceleration, approx. 9.81 $\mathrm{m} / \mathrm{s}^{2}$

$p \quad$ Pressure, $\mathrm{Pa}$

$p_{f} \quad$ Pressure drop due to flow friction, $\mathrm{Pa}$

$Q \quad$ Volumetric flow rate, $\mathrm{m}^{3} / \mathrm{s}$

$t \quad$ Time, $\mathrm{s}$

$V \quad$ Volume, $\mathrm{m}^{3}$

$v \quad$ Mean velocity over a channel crosssectional area, $\mathrm{m} / \mathrm{s}$

$X \quad$ Mass fraction of a fluid component, 1

$x \quad$ Axial spatial coordinate (along the fluid channel), $m$

$z \quad$ Vertical spatial coordinate, $\mathrm{m}$

Greek letters

$\rho \quad$ Density, $\mathrm{kg} / \mathrm{m}^{3}$

\section{References}

[1] Skoglund, T., Simulation of Liquid Food Processes in Modelica. Proceedings of the $3^{\text {rd }}$ International Modelica Conference 2003, 5158. Linköping, Sweden, November 3-4, 2003, Organized by the Modelica Association and Linköping University, Sweden. Available at www.modelica.org.

[2] Skoglund, T. and Dejmek P., A model library for dynamic simulation of liquid food process lines. Proceedings of FOODSIM 2006, 5-12, Naples, Italy, June 15-17, 2006, Organized by EUROSIS.

[3] Skoglund, T. and Dejmek, P. A dynamic object-oriented model for efficient simulation of fluid dispersion in turbulent flow with varying fluid properties. Chemical Engineering Science, 2007, 62, pp. 21682178.

[4] Skoglund, T. and Dejmek, P., Fuzzy traceability - A process simulation derived extension of the traceability concept in continuous food processing. Trans IChem
Part C, Food and Bio products Processing, 2007, 185, pp. 1-6.

[5] Skoglund, T., Dynamic Modelling and Simulation of Liquid Food Process Lines. Doctoral thesis, Department of Food Technology, Engineering and Nutrition, Faculty of Engineering, LTH, Lund University, Lund, Sweden, 2007. (ISBN 97891-976695-1-1)

[6] Modelica Association, http://www.modelica.org

[7] Dynasim AB, http://www.dynasim.se

[8] Skoglund, T. and Dejmek, P., A dynamic object-oriented model for efficient simulation of microbial reduction in dispersed turbulent flow. Journal of Food Engineering, 2008, 86, pp. 358-369.

[9] Eborn, J. On Model Libraries for Thermohydraulic Applications. Doctoral thesis, Department of Automatic Control, Faculty of Engineering, LTH, Lund University, Lund, Sweden, 2001.

[10] Tummescheit, H., Design and Implementation of Object-Oriented Model Libraries using Modelica, Doctoral thesis, Faculty of Engineering, LTH, Lund University, Lund, Sweden, 2002.

[11] Casella, F. and Leva, A., Modelica open library for power plant simulation: design and experimental validation. Proceedings of the $3^{\text {rd }}$ International Modelica Conference 2003. Linköping, Sweden, November 3-4, 2003, Organized by the Modelica Association and Linköping University, Sweden. Available at www.modelica.org.

[12] Gäfvert, M., Skoglund, T., Tummescheit H., Windahl, J., Wikander, H., Reuterswärd, P., Real-Time HWIL Simulation of Liquid Food Process Lines. Proceedings of the $6^{\text {th }}$ International Modelica Conference 2008, 709-715. Bielefeld, Germany, March 3-4, 2008, Organized by the Modelica Association and University of Applied Sciences, Bielefeld, Germany. Available at www.modelica.org. 